\title{
A Systematic Review and Meta-Analysis of Comparison of Outcomes of Robot-Assisted versus Open Partial Nephrectomy in Clinical T1 Renal Cell Carcinoma Patients
}

\author{
Yixiu Ni Xiaohua Yang \\ Department of Urology, the Second Affiliated Hospital (Jiande Branch), Zhejiang University School of \\ Medicine, Zhejiang, Hangzhou, China
}

\section{Keywords}

Renal cell carcinoma $\cdot$ Partial nephrectomy $\cdot$ Robotic surgery $\cdot$ Open surgery $\cdot$ Meta-analysis

\begin{abstract}
Introduction: Robot-assisted partial nephrectomy (RAPN) has been increasingly used for renal cell carcinoma in recent years. But the advantages of RARN over open partial nephrectomy (OPN) are still controversial. Methods: We searched the articles between 1997 and 2021 in PubMed, Web of Science, Cochrane Library, and EMbase databases. The parameters were perioperative outcomes including operating time (OT), warm ischemic time (WIT), estimated blood loss (EBL), positive surgical margin (PSM), preoperative and postoperative estimated glomerular filtration rate (eGFR), length of stay (LOS), and intraoperative and postoperative complications. Stata 13.0 software was used for the meta-analysis. Results: Seven studies with 2,646 patients $(1,285$ in RAPN vs. 1,361 in OPN) were included in the analysis. There were no significant differences in OT (WMD [95\% confidence interval $(\mathrm{Cl})]$ : $0.14[-0.33,0.61], p=0.570)$; WIT (WMD [95\% Cl]:0.28 [-0.13, 0.69], $p=0.187$ ); PSM (odds ratio [OR] [95\% Cl]: $1.04[0.37,2.94], p=0.944) ;$ preoperative eGFR
\end{abstract}

Karger@karger.com www.karger.com/uin

Karger ${ }^{\prime \prime} \div$

GOPEN ACCESS
C 2022 The Author(s).

Published by S. Karger AG, Basel

This article is licensed under the Creative Commons AttributionNonCommercial 4.0 International License (CC BY-NC) (http:// www.karger.com/Services/OpenAccessLicense). Usage and distribution for commercial purposes requires written permission.
(OR [95\% Cl]: $0.11[-0.01,0.23], p=0.071)$; postoperative eGFR (OR [95\% Cl]: $-0.11[0.27,0.04], p=0.159)$; and intraoperative complications (OR [95\% Cl]: 0.13 [0.02, 1.04], $p=$ 0.055 ) between 2 groups. But there were still less EBL (WMD [95\% Cl]: $-0.67[-1.07,-0.28], p=0.001)$, shorter LOS (WMD [95\% Cl]: $-1.09[-1.86,-0.32], p=0.005)$ and fewer postoperative complications (OR $[95 \% \mathrm{Cl}]$ : $0.51[0.38,0.68], p=$ 0.000). Conclusions: Compared with OPN, RAPN appears to achieve partly similar short-term functional outcomes. Meanwhile, some results are inconsistent with previous studies which seem to show that tumor type is also an important factor in comparison between RAPN and OPN, but the analysis is not carried out due to lack of complete data. Therefore, more high-quality random controlled trials are acquired.

(c) 2022 The Author(s).

Published by S. Karger AG, Basel

\section{Introduction}

Renal cell carcinoma (RCC) is the third most common urological cancer, and its incidence and detection rate have been increasing with the improvement and wide application of diagnostic tools in recent years $[1,2]$. Al- 
Fig. 1. Systematic reviews and meta-analysis flow diagram of studies identified, included and excluded.

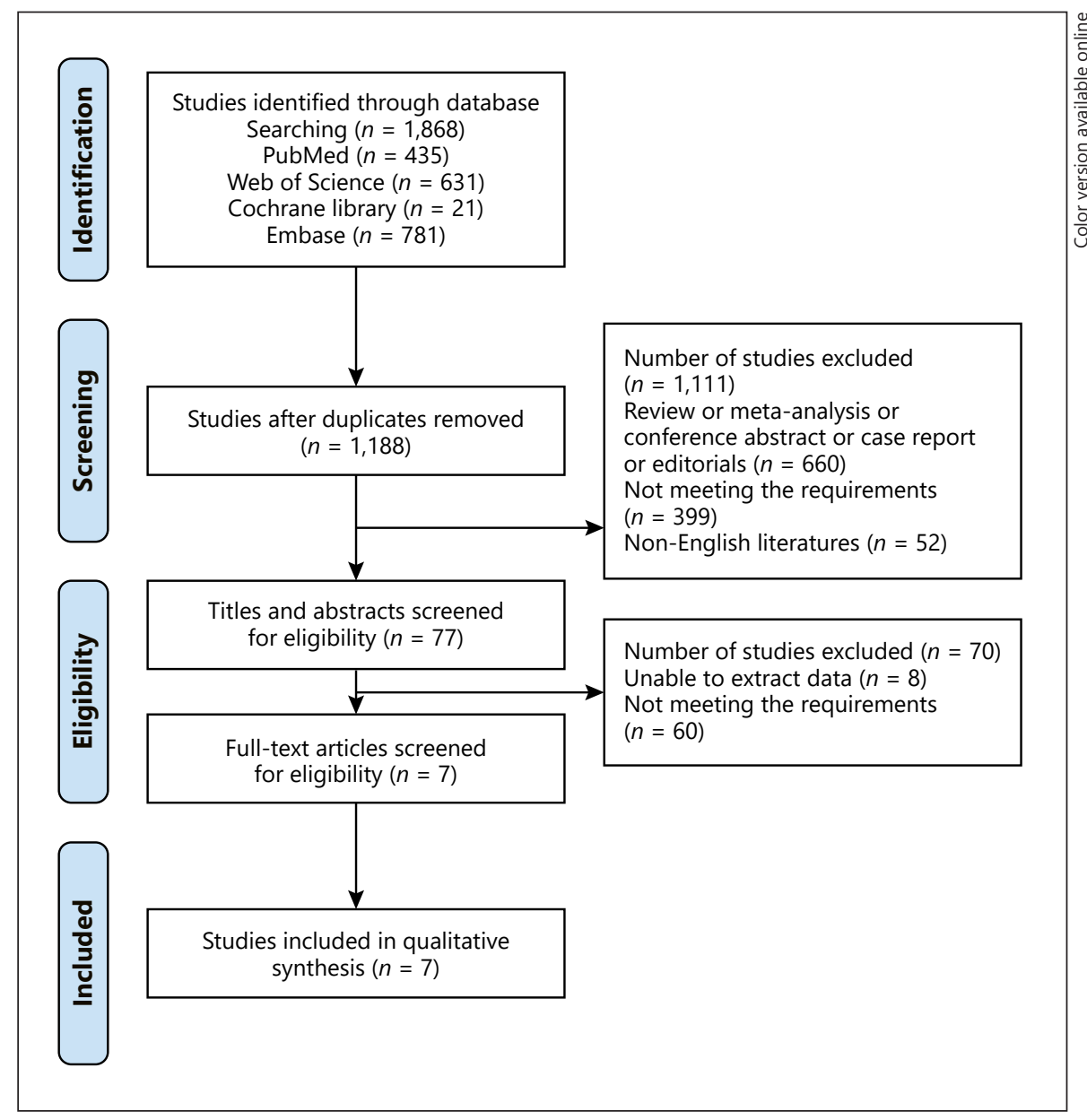

though new therapies have been developed, partial nephrectomy (PN) is still the "gold standard" for the localized cT1 RCCs [3]. However, PN is not fully utilized compared with radical nephrectomy $(\mathrm{RN})[4,5]$, although $\mathrm{PN}$ has been proven to be equivalent to $\mathrm{RN}$ in oncology for small renal masses [6]. In addition, the standard operation approach for $\mathrm{PN}$ is also disputed $[7,8]$. The one of possible reasons is relevant learning curve. For instance, laparoscopic PN has a steep learning curve, which restricts the use of urologists [9]. But the emergence of robot-assisted PN (RAPN) apparently reduced the learning curve $[10,11]$.

Previously, compared with laparoscopic PN, many studies have already verified the significant advantages of RAPN $[10,11]$. On the other hand, a meta-analysis also confirmed that RAPN appears to have lower morbidity and achieves similar short-term functional outcomes compared with open PN (OPN), but research objects are not limited to RCC patients [12]. Interestingly, a current meta-analysis has shown that RARN is not superior to LRN in patients with RCC in terms of perioperative outcomes [13]. Considering this situation, we aim to determine which surgical method is more suitable for patients with cT1 RCC through meta-analysis and systematic review.

\section{Materials and Methods}

Search Strategy

This systematic review and meta-analysis were performed in adherence to Preferred Reporting Items for Systematic Reviews and Meta-Analysis criteria [14]. We used the search terms: "robotassisted" OR "robotic-assisted" OR "robotic" OR "robot" OR "open"OR "partial nephrectomy" OR "renal cell carcinoma," and searched the articles which published between 1997 and 2021 in PubMed, Web of Science, Cochrane Library, and EMbase databases. The search was limited to publish in English. Two authors independently evaluated each included study and any differences or debates were resolved by consensus. 


\section{Inclusion/Exclusion Criteria}

Studies were included if they satisfied 4 criteria: (1) study type was randomized controlled trial, cohort study, or case-control study; (2) studies performed in adults diagnosed with T1 RCC; (3) studies comparing RAPN with OPN; and (4) at least, evaluation of one of the perioperative outcomes such as operative time (OT), warm ischemic time (WIT), estimated blood loss (EBL), postoperative length of hospital stay (LOS), preoperative estimated glomerular filtration rate (eGFR), postoperative eGFR, positive surgical margin (PSM), intraoperative complications and postoperative complications. Exclusion criteria were as follows: (1) no comparison performed between RAPN and OPN/LRN and OPN; (2) patients with benign and bilateral tumors; (3) patients with solitary kidney; (4) reviews, editorials, letters to editors, comments, case reports, unpublished articles and conference abstracts; and (5) studies with unavailable data.

\section{Data Extraction}

Data extraction was conducted independently by 2 authors, and discrepancies were resolved by negotiation. The following data were extracted from each study: first author, year of publication, region, time period of RAPN/OPN, institution, number of surgeons, study design, number of patients, age, sex, body mass index, tumor size, clinical stage, pathological stage and outcomes (including OT, WIT, EBL, LOS, preoperative eGFR, postoperative eGFR, PSM, intraoperative complications, and postoperative complications). If continuous variables in the article were expressed as median (interquartile range), it would be converted to mean \pm standard deviation [15].

\section{Risk-of-Bias Assessments}

The Risk of Bias in Non-Randomized Studies-of Interventions tool [16] was used to evaluate publication bias and assessed this bias according to seven domains: confounding bias, selection bias, bias in measurement classification of interventions, bias due to deviations from intended interventions, bias due to missing data, bias in measurement of outcomes, and bias in selection of the reported result.

\section{Quality Assessment}

All included studies were evaluated using the Newcastle-Ottawa scale [17], and the quality of studies was evaluated by measuring 3 aspects of the study design: patient selection, comparability of the study groups, and assessment of outcomes. Only when the study had a score of $\geq 6$ could it be considered as high quality, otherwise, the study was indicated as low quality.

\section{Statistical Analysis}

The meta-analysis was performed on Stata v.13.0 software (Stata Corp, College Station, TX, USA). Dichotomous and continuous variables were pooled as weighted mean difference (WMD) and odds ratio (OR), respectively. 95\% Confidence intervals (CIs) were used to assess all data. The degree of heterogeneity was measured by the I2 statistic, when $I^{2}>50 \%$, heterogeneity was considered significant statistically. The data were analyzed by a random-effect model; otherwise, a fixed-effects model was used. All statistical tests were performed two-sided, and $p \leq 0.05$ was considered statistically significant. Subgroup analysis was also carried out according to study design, setting, number of sample, and region. Sensitivity analysis was performed by omitting individual studies

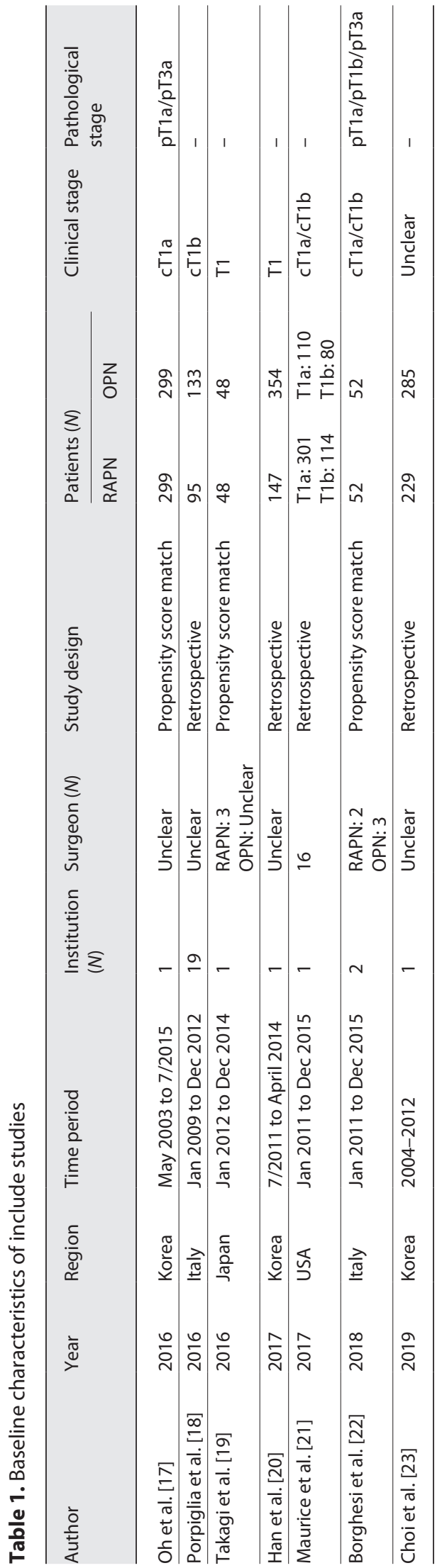


Table 2. Quality assessment of included studies

\begin{tabular}{|c|c|c|c|c|c|c|c|c|c|c|}
\hline \multirow[t]{2}{*}{ Study } & \multicolumn{4}{|c|}{ Selection } & \multicolumn{2}{|c|}{ Comparability } & \multicolumn{3}{|c|}{ Exposure } & \multirow[t]{2}{*}{ Total points } \\
\hline & REC & SNEC & $\mathrm{AE}$ & DO & SC & $\mathrm{AF}$ & $\mathrm{AO}$ & FU & AFU & \\
\hline Oh et al. [17] & 1 & 1 & 1 & 1 & 1 & & 1 & 1 & 1 & $8^{*}$ \\
\hline Porpiglia et al. [18] & 1 & 1 & 1 & 1 & 1 & & 1 & 1 & 1 & $8^{*}$ \\
\hline Takagi et al. [19] & 1 & 1 & 1 & 1 & 1 & & 1 & & 1 & $7^{*}$ \\
\hline Han et al. [20] & 1 & 1 & 1 & 1 & 1 & & 1 & & & 6 \\
\hline Maurice et al. [21] & 1 & 1 & 1 & 1 & 1 & & 1 & & & 6 \\
\hline Borghesi et al. [22] & 1 & 1 & 1 & 1 & 1 & & 1 & & & 6 \\
\hline Choi et al. [23] & 1 & 1 & 1 & 1 & 1 & & 1 & 1 & 1 & $8^{*}$ \\
\hline
\end{tabular}

REC, representativeness of the cohort; SNEC, selection of the none posed cohort; $A E$, ascertainment of exposure; DO, demonstration that outcome of interest was not present at start of study; SC, study controls most important factors; AF, study controls for other important factors; AO, assessment of outcome; FU, follow-up long enough for outcomes to occur ("long enough" is defined as 1 year); AFU, adequacy of follow-up of cohort ( $\geq 80 \%)$. * Means that the study is satisfied the item, the quality score $\geq 7$ points was ranked as high.

Table 3. Patient demographics and tumor characteristics of include studies

\begin{tabular}{|c|c|c|c|c|c|c|c|c|c|}
\hline \multirow[t]{2}{*}{ Outcomes } & \multirow{2}{*}{$\begin{array}{l}\text { Studies } \\
(N)\end{array}$} & \multicolumn{2}{|c|}{ Patients $(N)$} & \multirow[t]{2}{*}{ WMD or OR $[95 \% \mathrm{CI}]$} & \multirow[t]{2}{*}{$p$ value* } & \multicolumn{4}{|c|}{ Heterogeneity } \\
\hline & & RAPN & OPN & & & $x^{2}$ & df & $p$ value ${ }^{*}$ & $R^{2}, \%$ \\
\hline Age & 8 & 1,285 & 1,361 & $-0.212[-0.291,-0.132]$ & 0.000 & 3.13 & 7 & 0.873 & 0.0 \\
\hline Sex (male) & 8 & 1,285 & 1,361 & $0.043[-0.085,0.172]$ & 0.508 & 16.35 & 7 & 0.022 & 57.2 \\
\hline BMI & 8 & 1,285 & 1,361 & $0.936[0.828,1.059]$ & 0.295 & 3.87 & 7 & 0.795 & 0.0 \\
\hline Tumor size & 5 & 1,190 & 1,228 & $-0.172[-0.273,-0.072]$ & 0.001 & 2.11 & 4 & 0.716 & 0.0 \\
\hline R.E.N.A.L score & 4 & 614 & 596 & $0.04[-0.082,0.162]$ & 0.521 & 9.07 & 3 & 0.028 & 66.9 \\
\hline
\end{tabular}

* Statistically significant results are shown in bold.

one by one for some outcomes such as OT, WIT, EBL, and LOS. Since there were less than 10 studies in this meta-analysis, funnel plot was not used to assess publication bias.

\section{Results}

\section{Study Characteristics}

As shown in Figure 1, 7 eligible studies were finally included with a total of 2,646 patients ( 1,285 patients in the RAPN and 1,361 in OPN) [17-23]. Among the 7 studies, there were 3 propensity score matched $[17,19,22]$ and 4 retrospective studies $[18,20,21,23]$. Five studies were single-center $[17,19-21,23]$ and 2 studies were multicenter $[18,22]$. It is pity that no studies were single surgeon and most of them were unclear. All the studies mentioned clinical stage except for one study [23]. Among them, 2 studies $[17,18]$ included only $\mathrm{cT} 1 \mathrm{a}$ and $\mathrm{cT} 1 \mathrm{~b}$ RCC patients, respectively. The clinical stage of RCC patients in 2 studies $[19,20]$ was not provided in detail. The
2 studies [21,22] included cT1a and cT1b RCC patients, but one of them separated the 2 populations [21], and the other was the opposite [22]. In addition, pathological stage was performed in only 2 studies [17, 22]. The characteristics of included studies were presented in Table 1, and the quality assessment of included studies was presented in Table 2.

\section{Demographics and Tumor Characteristics Variable}

As shown in Table 3 and online supplementary Figure S1 (see www.karger.com/doi/10.1159/000521881 for all online suppl. material), there were no significant differences between the 2 groups in terms of sex (male: OR [95\% CI]: $0.043[-0.085,0.172] ; p=0.508)$, body mass index (WMD [95\% CI]: 0.936 [0.828, 1.059]; $p=0.295$ ) and R.E.N.A.L score (WMD [95\% CI]: 0.04 [-0.082, $0.162] ; p=0.521)$, but there were significant differences between the 2 groups in terms of age (WMD [95\% CI]: $-0.212[-0.291,-0.132] ; p=0.000)$ and tumor size (WMD [95\% CI]: $-0.172[-0.273,-0.072] ; p=0.001)$. 


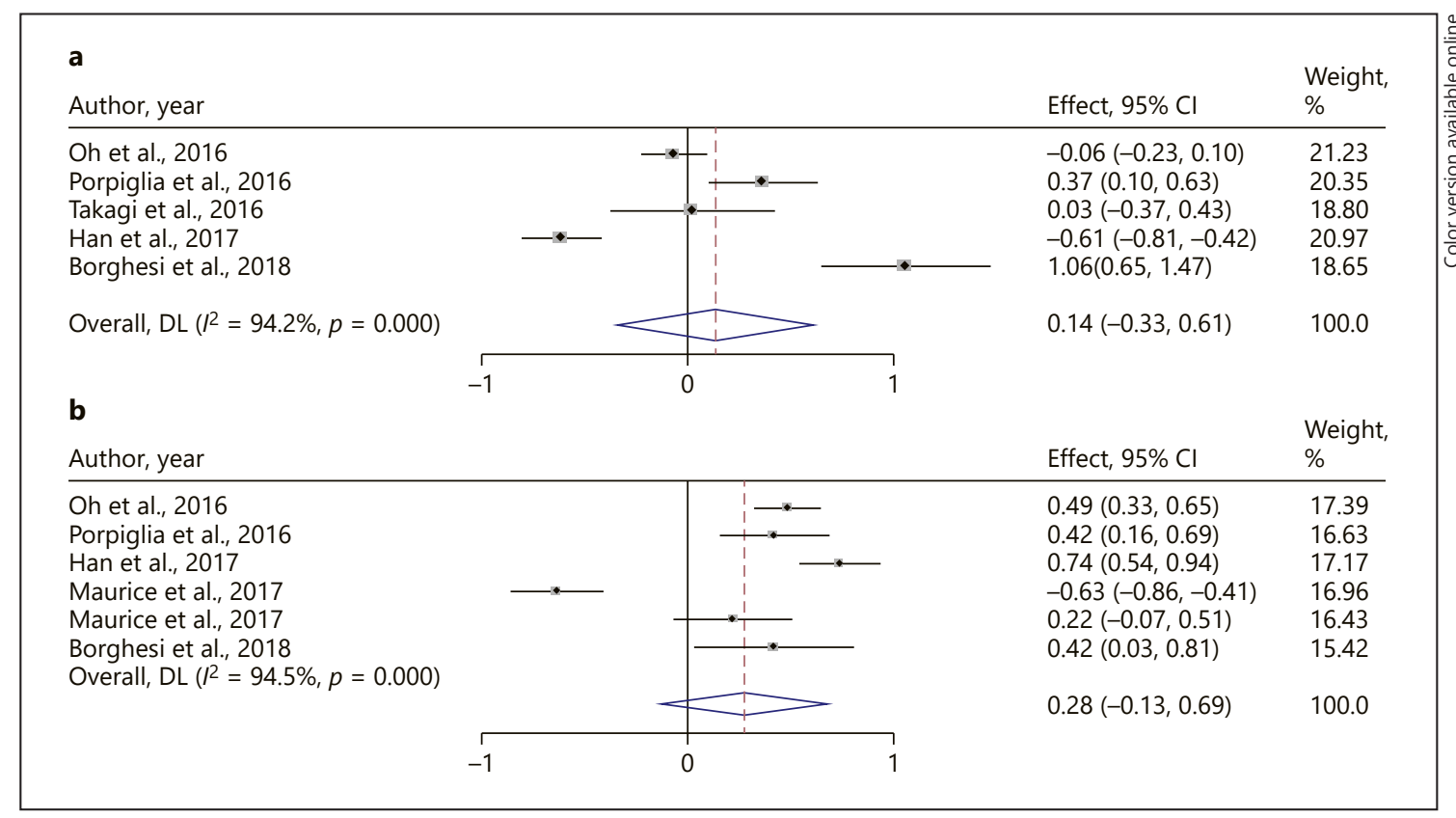

Fig. 2. Forest plots evaluating the OT and the WIT between RAPN and OPN: the OT (a); WIT (b). Note: weights are from random-effects model.

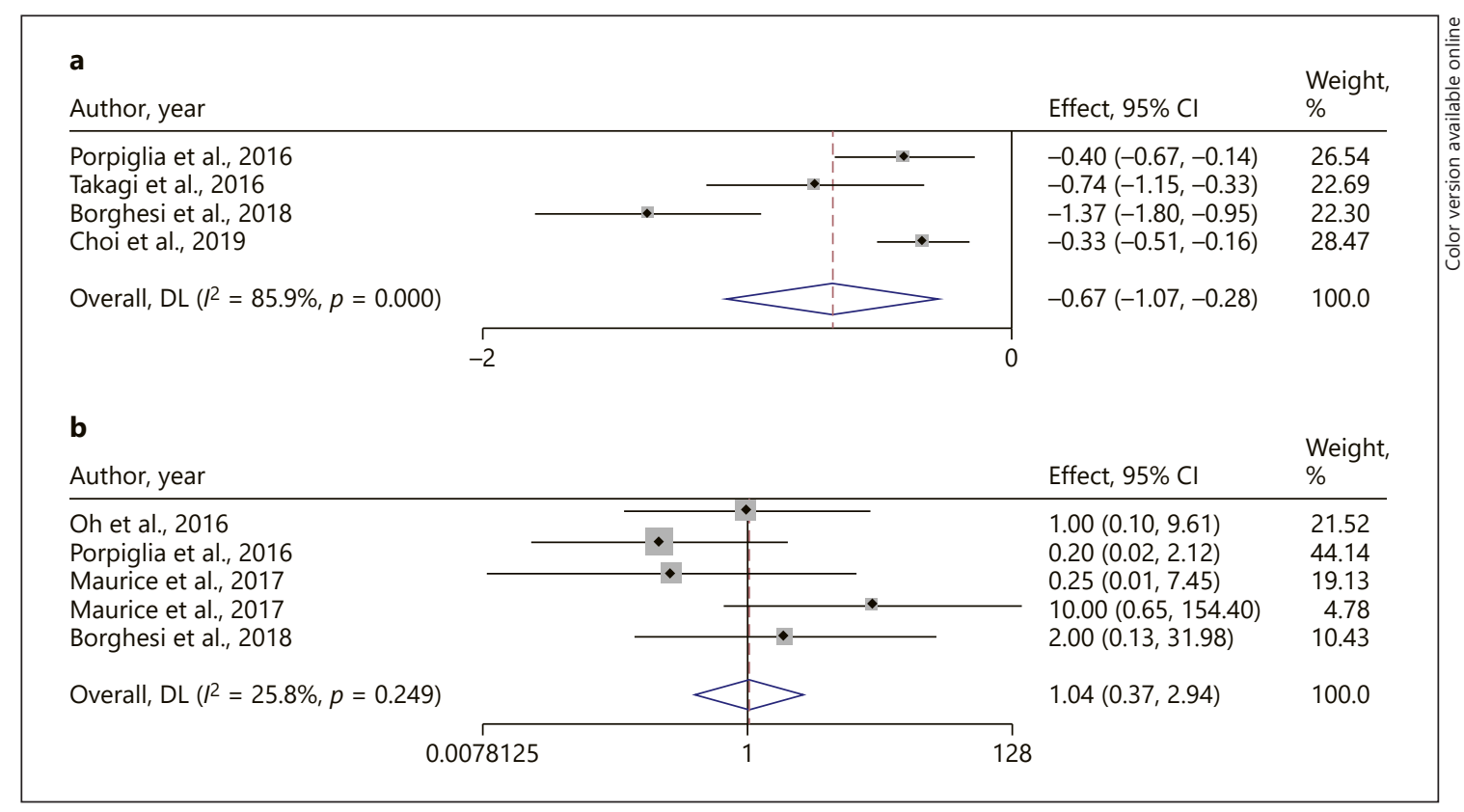

Fig. 3. Forest plots of studies evaluating the EBL and the PSM between RAPN and OPN: EBL (a); PSM (b). Note: weights are from random-effects model.

\section{Meta-Analysis Results}

Operative Time

Five studies were included in the analysis of OT, and total of 1,527 patients (641 in RAPN vs. 886 in OPN) [17$20,22]$. A random-effect model was used to analyze the data $\left(I^{2}=94.2 \%\right)$. The results indicated no significant differences for OT between RAPN and OPN (WMD [95\% $\mathrm{CI}]: 0.14[-0.33,0.61], p=0.570)$, although there was a high heterogeneity (Fig. 2a). 


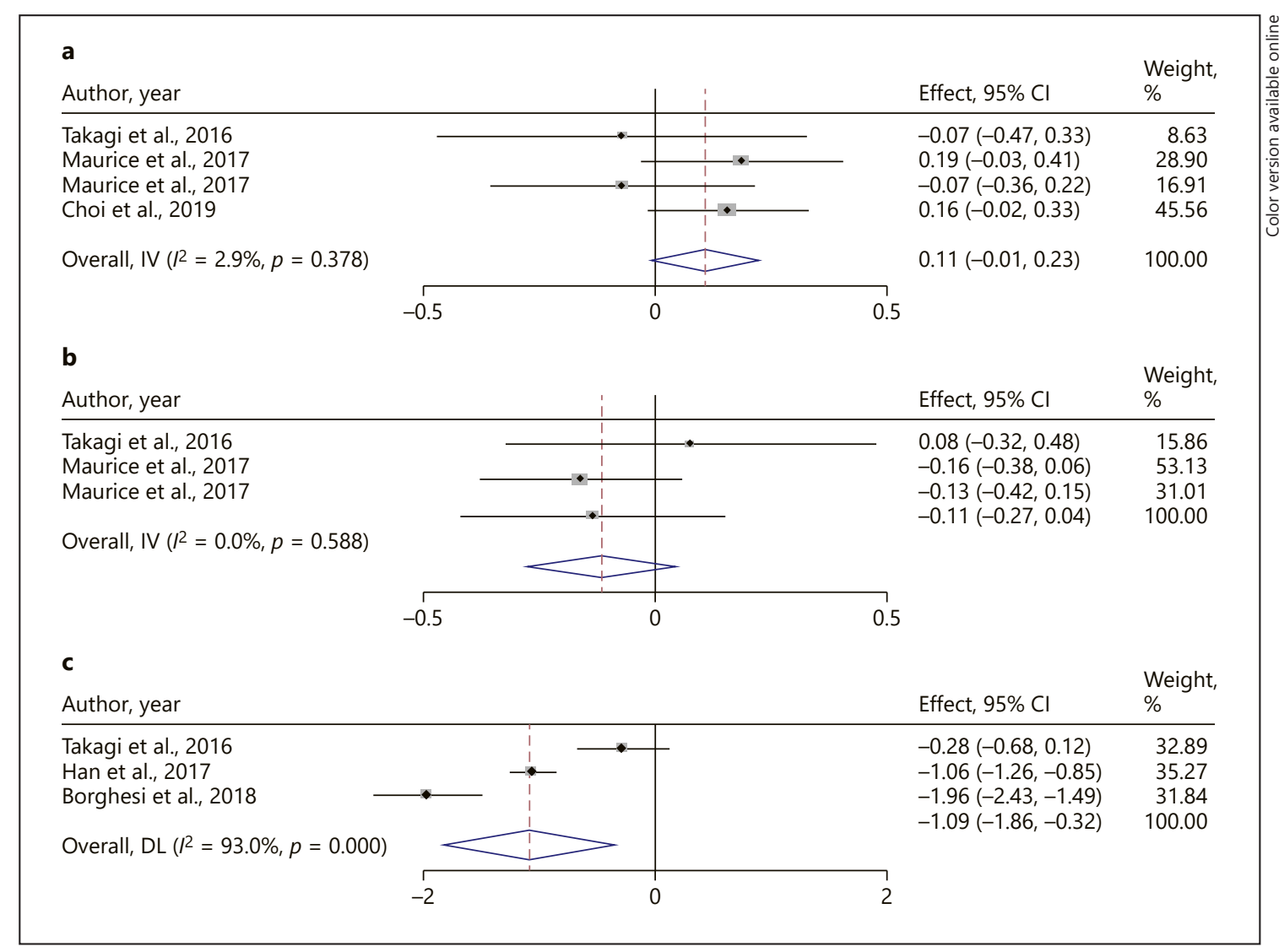

Fig. 4. Forest plots of evaluating the preoperative eGFR, postoperative eGFR, and the LOS between RAPN and OPN: preoperative eGFR (a); postoperative eGFR (b); postoperative LOS (c). Note: weights are from randomeffects model.

Warm Ischemic Time

The data of WIT were obtained from 5 studies and including 2023 patients (999 in RAPN vs. 1,024 in OPN) $[17,18,20-22]$. There was also no significant difference between RAPN and OPN in the pooled analysis (randomeffect model: $I^{2}=94.5 \%$, WMD [95\% CI]: $0.28[-0.13$, 0.69 ], $p=0.187$ ) (Fig. 2b).

Estimated Blood Loss

Four studies were included in the meta-analysis [18, $19,22,23$ ], and totaling 942 patients (RAPN vs. OPN: 424 vs. 518). Due to a great heterogeneity, a random-effect model was used $\left(I^{2}=85.9 \%\right)$. The pooled analysis indicated EBL in RAPN was less than that in OPN (WMD [95\% CI]: $-0.67[-1.07,-0.28], p=0.001$ ) (Fig. 3a).

\section{Positive Surgical Margin}

1,522 patients were analyzed in 4 studies [17, 18, 21, 22]. PSM was reported in $27 / 852$ of patients who underwent RAPN and 24/670 of patients who underwent OPN.
The data showed that RAPN offered a comparable PSM to OPN (fixed effect model: $I^{2}=25.8 \%$, OR [95\% CI]: 1.04 $[0.37,2.94], p=0.944)$ (Fig. 3b).

Preoperative and Postoperative Estimated Glomerular Filtration Rate

Three studies were included in the analysis of preoperative eGFR $[19,21,23]$, and two were included in the analysis of postoperative eGFR $[19,21]$. Among them, a total of 1,215 patients in preoperative eGFR (692 in RAPN vs. 523 in OPN), and a total of 701 patients in postoperative eGFR (463 in RAPN vs. 238 in OPN). The results were similar between the 2 groups, whatever were preoperative eGFR or postoperative eGFR, and low heterogeneity were found in the two eGFRs (preoperative eGFR: fixed effect model: $I^{2}=2.9 \%$, OR [95\% CI]: $0.11[-0.01$, 0.23 ], $p=0.071$; postoperative eGFR: fixed effect model: $I^{2}=0.0 \%$, OR [95\% CI]: $\left.-0.11[0.27,0.04], p=0.159\right)$ (Fig. 4a-b). 


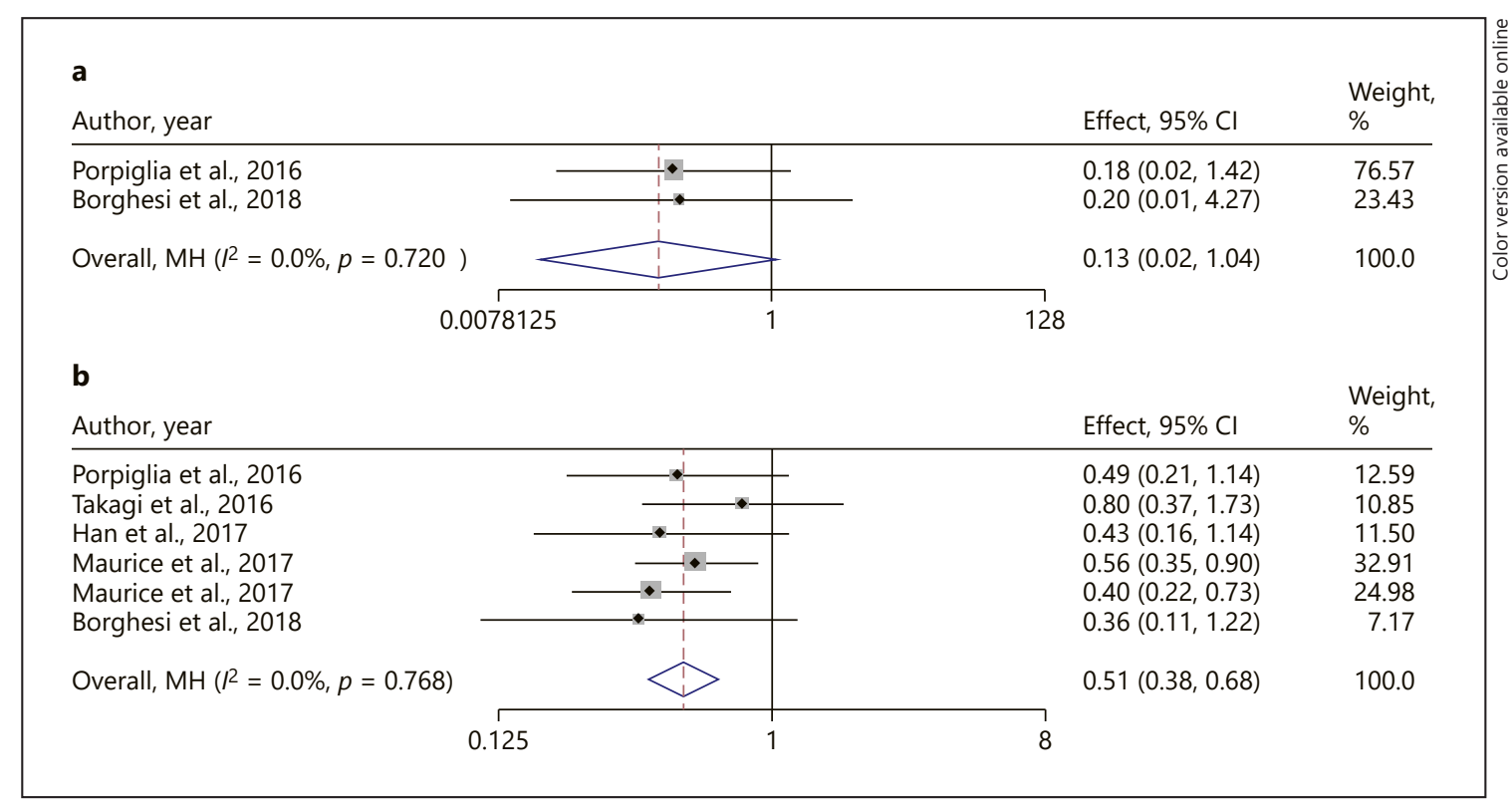

Fig. 5. Forest plots of evaluating the intraoperative and postoperative complications between RAPN and OPN: intraoperative complications (a); postoperative complications (b). Note: weights are from Mantel-Haenszel model.

Postoperative Length of Hospital Stay

There were 3 studies were included in reporting LOS, involving 701 patients, of which 247 underwent RAPN and 454 underwent OPN. The data showed that the patients who underwent RAPN had a shorter postoperative LOS than those who underwent OPN (random-effect model: $I^{2}=93.0 \%$, WMD [95\% CI]: $-1.09[-1.86,-0.32]$, $p=0.005)$ (Fig. 4c).

\section{Intraoperative and Postoperative Complications}

The illustrations of intraoperative and postoperative complications were set in Figure 5. There was no significant difference in intraoperative complications between the two approaches (fixed effect model: $I^{2}=0.0 \%$, OR [95\% CI]: 0.13 [0.02, 1.04], $p=0.055$ ) (Fig. 5a), but the postoperative complications were dramatically fewer in RAPN compared to OPN (fixed effect model: $I^{2}=$ $0.0 \%$, OR [95\% CI]: $0.51[0.38,0.68], p=0.000)$ (Fig. 5b).

\section{Subgroup Analysis}

Subgroup analysis of OT was shown in Table 4 and online supplementary Figure S2. The findings of no significant difference for OT were consistent in all subgroup analysis except for multicenter and Europe subgroup. Subgroup differences were found in setting and region.

Comparison of Robot-Assisted versus

Open Partial Nephrectomy in cT1 RCC
Sensitivity Analysis and Publication Bias

Although the included studies had a high quality (all studies had a 6 score or higher), some parameters such as OT, WIT, EBL, and LOS were still highly heterogeneous. So, it is necessary that a sensitivity analysis was performed to improve the reliability of the analysis of these parameters. Deleting studies one by one to recalculate the combined average difference. After deleting any one study, all new pooled mean differences remained unchanged (Fig. 6). Publication bias was assessed by the Risk of Bias in Non-Randomized Studies-of Interventions tool and suggested that all comparative studies had a moderate risk of bias [17-23].

\section{Discussion}

Many studies focused on the comparison of perioperative outcomes between RAPN and OPN [17, 19, 22], but there are not restrictions in the tumor types of patients (including malignant tumors (clear cell, papillary, and chromophobe) and benign tumors (angiomyolipoma [AML] and oncocytoma)). To the best of our knowledge, this is the first meta-analysis to report the comparative outcomes of RAPN versus OPN in patients with clinical T1 (cT1) RCC. Currently, several studies suggested that $\mathrm{PN}$ is considered as the suitable option for clinical T1 
Table 4. Subgroup analysis of OT

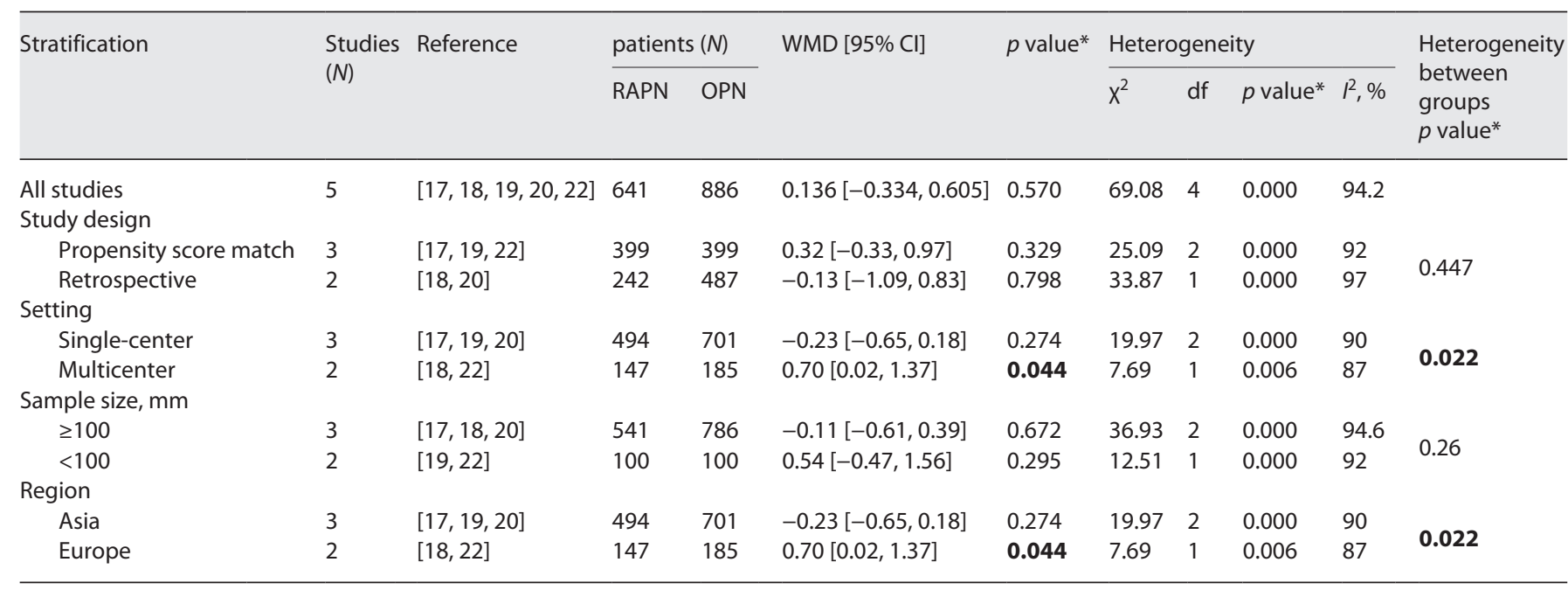

* Statistically significant results are shown in bold.

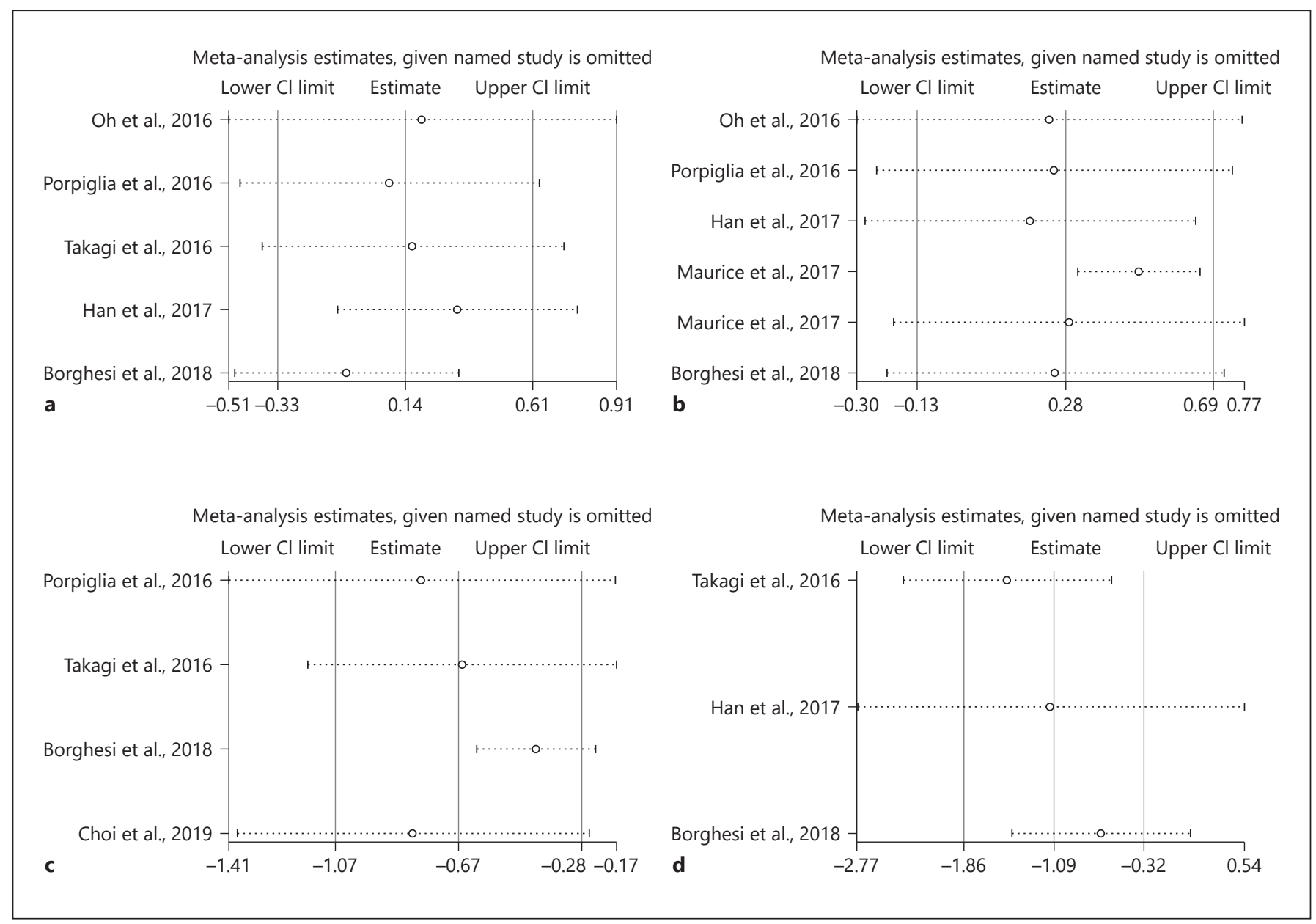

Fig. 6. Sensitivity analysis of studies reporting the 4 perioperative outcomes between RAPN and OPN: OT (a); WIT (b); $\operatorname{EBL}(\mathbf{c}) ; \operatorname{LOS}(\mathbf{d})$. 
RCC [24-26]. In this study, we indicated that there were no significant differences in terms of OT, WIT, PSM, preoperative eGFR, postoperative eGFR, and intraoperative complications. Hence, RAPN appears to be a safety and effective surgical treatment for patients with cT1 RCC, although only perioperative outcomes were compared in this meta-analysis.

In the meta-analysis conducted by Xia et al. [12], the analysis of pooled data showed that there is a longer OT and WIT in RAPN. Grivas et al. [27] also found the consistent results. However, our analysis found that OT and WIT are no significant difference between the 2 groups. It seemed to indicate that OT and WIT are related to the tumor types of patients. But considering the high heterogeneity $\left(I^{2}=94.2 \%\right)$, these results should be interpreted with caution. On the other hand, subgroup analysis showed that OT appears to be also related to the difference from setting and region, and it is interesting that all single-center studies are from Asia and multicenter studies are from Europe. In fact, OT may be related to the technical proficiency and experience of the surgeon, for example, the mean OT of robotic surgery of Takagi et al. [19] is nearly $1 \mathrm{~h}$ longer than that of Oh et al. [17]. Similarly, the WIT is related with ability to suture with RAPH, and the WIT of RAPN can be controlled to less than 20 $\mathrm{min}$, if it is operated by an experienced surgeon $[10,18$, 28]. Some initial experiences from a few studies which focused on the outcomes of RAPN for T1b renal lesions suggested that a significant higher WIT in T1b to T1a renal tumors $[29,30]$. In addition, the EBL and LOS in RAPH were less than that in OPN, which are consistent with previous studies $[19,22]$.

Our meta-analysis showed similar PSM rate, preoperative eGFR, and postoperative eGFR between RAPN and OPN groups. Although it is still controversial whether PSM will affect the long-term oncology results of patients with RCC, complete removal of the tumor and achieving a negative margin is undoubtedly one of the key goals of PN $[31,32]$. In terms of complications, we found that patients undergoing RAPN had a lower rate of postoperative complications, while intraoperative complications had no significance difference between the 2 groups. Similar findings were also supported by the recent metaanalysis $[12,27]$.

When it comes to some parameters, such as OT and WIT, which were different from the results of previous studies, it is confirmed that tumor types might be an important factor. However, due to lack of complete data in included studies, we could not carry out subgroup analysis of clinical and pathological stages.

Comparison of Robot-Assisted versus

Open Partial Nephrectomy in cT1 RCC
In fact, Cacciamani et al. [33] recently report a study protocol about comparing robotic versus open urological oncological surgery, and point out that the comparison between them should focus on five key questions: penetrance in the field, perioperative data, procedural morbidity, oncological outcomes, functional outcomes, and financial cost. But it is regrettable that the outcomes of cost are not offered by included studies. Therefore, in future research, cost is designed as one of the parameters, which can prove more objectively and comprehensively that RAPN is superior to OPN. However, cost variations may depend on the characteristics of the hospital (such as size, geography, teaching status, and profit status) [34, 35], and these potential confounding factors lead to uncertainty in the cost results.

In addition, it is worth noting that age and tumor size had significant differences between the 2 groups. After a series of analyses, we found that patients of RAPN group (mean age ranged from 51.7 to 61.35 years old) were younger than OPN group (mean age ranged from 53.25 to 62.77 years old) and mean tumor size of RAPN group (ranged from 2.5 to 4.96 years old) was smaller than OPN group (ranged from 2.8 to $5 \mathrm{~cm}$ ). However, it is necessary that demographic data should be as comparable as possible to ensure that the influence of confounding factors on the study was minimized and the results were more reliable. Hence, researchers need to pay more attention to the comparability of demographic data in research design.

However, there are some limitations in the present analysis. First, most of the included studies were the retrospective analysis, and some studies were small sample size. Second, the learning curve of the surgeons was not accounted for before conducting the analyses, and it is worth mentioning that most of the studies declared the surgeons were beyond learning curve. Third, heterogeneities between the studies for OT, WIT, EBL, and LOS were relatively high. The possible explanation is that all of them are continues variables; on the contrary, categorical variables tend to have low heterogeneities. But this cannot explain all the data, after all, the heterogeneity of preoperative eGFR and postoperative eGFR is not high. Fourth, due to incomplete data, it is difficult to get the ASA score with statistical significance so that the quality of meta-analysis was limited. Finally, a cost outcomes was not included due to lack of data. Indeed, cost is the one of main factors which might restrict the application of robotics. 


\section{Conclusion}

Our meta-analysis suggests that RAPN may be more suitable for the patients with cT1 RCC, as it offers less EBL, shorter LOS, and fewer postoperative complications. Meanwhile, some results are inconsistent with previous studies showing that tumor type may be an important factor in comparison between RAPN and OPN. However, the analysis is not carried out due to lack of complete data. Therefore, random controlled trials with well-designed, large sample size, and long-term fellow-up are still needed, and tumor type should need to be taken into account.

\section{Statement of Ethics}

Ethical approval was not required for this study in accordance with local/national guidelines.

\section{Conflict of Interest Statement}

The authors have no conflicts of interest to declare.

\section{Funding Sources}

This manuscript did not receive any funding.

\section{Author Contributions}

Y.N. planned and designed the research; XY. tested the feasibility of the study; Y.N. wrote the manuscript; all the authors approved the final version of the manuscript.

\section{Data Availability Statement}

All data generated or analyzed during this study are included in this. Further enquiries can be directed to the corresponding author.

\section{References}

1 Capitanio U, Bensalah K, Bex A, Boorjian SA, Bray F, Coleman J, et al. Epidemiology of renal cell carcinoma. Eur Urol. 2019;75(1):7484.

2 Ziegelmüller BK, Spek A, Szabados B, Casuscelli J, Clevert DA, Staehler M. Epidemiology and diagnostic assessment of small renal masses. Urologe A. 2018;57(3):274-9.

3 Asker AA, Addar A, Alghamdi M, Alawad S, Alharbi M, Hamri SB, et al. Partial nephrectomy, a comparison between different modalities: a tertiary care center experience. J Kidney Cancer VHL. 2021;8(2):34-9.

4 Liss MA, Wang S, Palazzi K, Jabaji R, Patel N, Lee HJ, et al. Evaluation of national trends in the utilization of partial nephrectomy in relation to the publication of the American Urologic Association guidelines for the management of clinical T1 renal masses. BMC Urol. 2014; 14:101.

5 Liu JJ, Leppert JT, Maxwell BG, Panousis P, Chung BI. Trends and perioperative outcomes for laparoscopic and robotic nephrectomy using the national surgical quality improvement program (NSQIP) database. Urol Oncol. 2014;32(4):473-9.

6 Zeuschner P, Greguletz L, Meyer I, Linxweiler J, Janssen M, Wagenpfeil G, et al. Open versus robot-assisted partial nephrectomy: a longitudinal comparison of 880 patients over 10 years. Int J Med Robot. 2021;17(1):1-8.

7 Gettman MT, Blute ML, Chow GK, Neururer R, Bartsch G, Peschel R. Robotic-assisted laparoscopic partial nephrectomy: technique and initial clinical experience with DaVinci robotic system. Urology. 2004;64(5):914-8.
8 Winfield HN, Donovan JF, Godet AS, Clayman RV. Laparoscopic partial nephrectomy: initial case report for benign disease. J Endourol. 1993;7(6):521-6.

9 Porpiglia F, Volpe A, Billia M, Scarpa RM. Laparoscopic versus open partial nephrectomy: analysis of the current literature. Eur Urol. 2008;53(4):732-3; discussion 742-3.

10 Zargar H, Allaf ME, Bhayani S, Stifelman M, Rogers C, Ball MW, et al. Trifecta and optimal perioperative outcomes of robotic and laparoscopic partial nephrectomy in surgical treatment of small renal masses: a Multi-Institutional Study. BJU Int. 2015;116(3):40714.

11 Choi JE, You JH, Kim DK, Rha KH, Lee SH. Comparison of perioperative outcomes between robotic and laparoscopic partial nephrectomy: a systematic review and metaanalysis. Eur Urol. 2015;67(5):891-901.

12 Xia L, Wang X, Xu T, Guzzo TJ. Systematic review and meta-analysis of Comparative Studies reporting perioperative outcomes of robot-assisted partial nephrectomy versus open partial nephrectomy. J Endourol. 2017; 31(9):893-909.

13 Li J, Peng L, Cao D, Cheng B, Gou H, Li Y, et al. Comparison of perioperative outcomes of robot-assisted versus laparoscopic radical nephrectomy: a systematic review and metaanalysis. Front Oncol. 2020;10:551052.

14 Moher D, Liberati A, Tetzlaff J, Altman DG, PRISMA Group. Preferred reporting items for systematic reviews and meta-analyses: the PRISMA statement. BMJ. 2009;339:b2535.
15 Wan X, Wang W, Liu J, Tong T. Estimating the sample mean and standard deviation from the sample size, median, range and/or interquartile range. BMC Med Res Methodol. 2014;14:135.

16 Sterne JA, Hernán MA, Reeves BC, Savović J, Berkman ND, Viswanathan M, et al. ROBINS-I: a tool for assessing risk of bias in NonRandomised Studies of interventions. BMJ. 2016;355:i4919.

17 Oh JJ, Lee JK, Kim K, Byun SS, Lee SE, Hong SK. Comparison of the width of peritumoral surgical margin in open and robotic partial nephrectomy: a propensity score matched analysis. PLoS One. 2016;11(6):e0158027.

18 Porpiglia F, Mari A, Bertolo R, Antonelli A, Bianchi G, Fidanza F, et al. Partial nephrectomy in clinical T1b renal tumors: Multicenter Comparative Study of open, laparoscopic and robot-assisted approach (the RECORd project). Urology. 2016;89:45-51.

19 Takagi T, Kondo T, Tachibana H, Iizuka J, Omae K, Kobayashi H, et al. A propensity score-matched comparison of surgical precision obtained by using volumetric analysis between robot-assisted laparoscopic and open partial nephrectomy for T1 renal cell carcinoma: a retrospective non-randomized observational study of initial outcomes. Int Urol Nephrol. 2016;48(10):1585-91.

20 Han KS, Song GH, You D, Song C, Jeong IG, Hong JH, et al. Comparison of hand-assisted laparoscopic vs robot-assisted laparoscopic versus open partial nephrectomy in patients with T1 renal masses. J Endourol. 2017;31(4): 374-9. 
21 Maurice MJ, Ramirez D, Kara Ö, Malkoç E, Nelson RJ, Fareed K, et al. Optimum outcome achievement in partial nephrectomy for T1 renal masses: a contemporary analysis of open and robot-assisted cases. BJU Int. 2017; 120(4):537-43.

22 Borghesi M, Schiavina R, Chessa F, Bianchi L, La Manna G, Porreca A, et al. Retroperitoneal robot-assisted versus open partial nephrectomy for cT1 renal tumors: a matched-pair comparison of perioperative and early oncological outcomes. Clin Genitourin Cancer. 2018;16(2):e391-e396.

23 Choi SY, Jung H, You D, Jeong IG, Song C, Hong B, et al. Robot-assisted partial nephrectomy is associated with early recovery of renal function: comparison of open, laparoscopic, and robot-assisted partial nephrectomy using DTPA renal scintigraphy. J Surg Oncol. 2019; 119(7):1016-23.

24 Ljungberg B, Bensalah K, Canfield S, Dabestani S, Hofmann F, Hora M, et al. EAU guidelines on renal cell carcinoma: 2014 update. Eur Urol. 2015;67(5):913-24.
25 Campbell S, Uzzo RG, Allaf ME, Bass EB, Cadeddu JA, Chang A, et al. Renal mass and localized renal cancer: AUA guideline. J Urol. 2017;198(3):520-9.

26 Janssen MWW, Linxweiler J, Terwey S, Rugge $\mathrm{S}$, Ohlmann CH, Becker F, et al. Survival outcomes in patients with large $(\geq 7 \mathrm{~cm})$ clear cell renal cell carcinomas treated with nephronsparing surgery versus radical nephrectomy: results of a multicenter cohort with long-term follow-up. PLoS One. 2018;13(5):e0196427.

27 Grivas N, Kalampokis N, Larcher A, Tyritzis $\mathrm{S}$, Rha $\mathrm{KH}$, Ficarra V, et al. Robot-assisted versus open partial nephrectomy: comparison of outcomes. A systematic review. Minerva Urol Nefrol. 2019;71(2):113-20.

28 Ficarra V, Minervini A, Antonelli A, Bhayani S, Guazzoni G, Longo N, et al. A multicentre matched-pair analysis comparing robot-assisted versus open partial nephrectomy. BJU Int. 2014 Jun;113(6):936-41.

29 Petros F, Sukumar S, Haber GP, Dulabon L, Bhayani S, Stifelman M, et al. Multi-institutional analysis of robot-assisted partial nephrectomy for renal tumors $>4 \mathrm{~cm}$ versus $\leq 4$ $\mathrm{cm}$ in 445 consecutive patients. J Endourol. 2012;26(6):642-6.
30 Patel MN, Krane LS, Bhandari A, Laungani RG, Shrivastava A, Siddiqui SA, et al. Robotic partial nephrectomy for renal tumors larger than $4 \mathrm{~cm}$. Eur Urol. 2010;57(2):310-6.

31 Borghesi M, Brunocilla E, Schiavina R, Martorana G. Positive surgical margins after nephron-sparing surgery for renal cell carcinoma: incidence, clinical impact, and management. Clin Genitourin Cancer. 2013; 11(1):5-9.

32 Marszalek M, Carini M, Chlosta P, Jeschke K, Kirkali Z, Knüchel R, et al. Positive surgical margins after nephron-sparing surgery. Eur Urol. 2012;61(4):757-63.

33 Cacciamani GE, Gill K, Gill IS. Robotic versus open urological oncological surgery: Study Protocol of a systematic review and metaanalysis. BMJ Open. 2020;10(2):e036609.

34 Bai G, Anderson GF. Extreme markup: the fifty US hospitals with the highest charge-tocost ratios. Health Aff. 2015;34(6):922-8.

35 Balakrishnan AS, Hampson LA, Bell AM, Baghdanian AH, Baghdanian AA, Meng MV, et al. Evaluating the impact of surgical supply cost variation during partial nephrectomy on patient outcomes. Transl Androl Urol. 2021; 10(2):765-74. 